\section{Asymptomatic muscle metastasis from pulmonary adenocarcinoma}

A 62-year-old never-smoker woman was admitted with a 2-month history of progressive dyspnoea. Her CT scan showed a $3 \times 4 \mathrm{~cm}$ lung mass in the right upper lobe. Tumour biopsy revealed an adenocarcinoma. Integrated $\left[{ }^{18} \mathrm{~F}\right]$ fluorodeoxyglucose
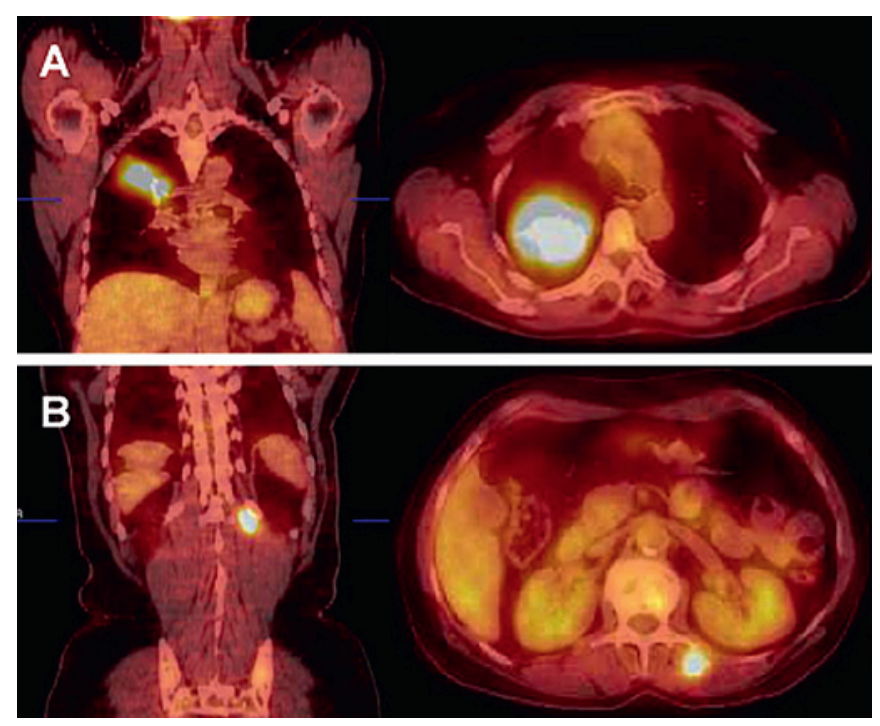

Figure 1 (A) Coronal and axial views of integrated $\left[{ }^{18} \mathrm{~F}\right]$ fluorodeoxyglucose positron emission tomography (FDG-PET)/CT scan showing abnormal FDG uptake in the right upper lobe lung adenocarcinoma. (B) At the level of muscle metastasis, fused images demonstrate pathologically increased FDG focal uptake in the left paraspinal muscle.

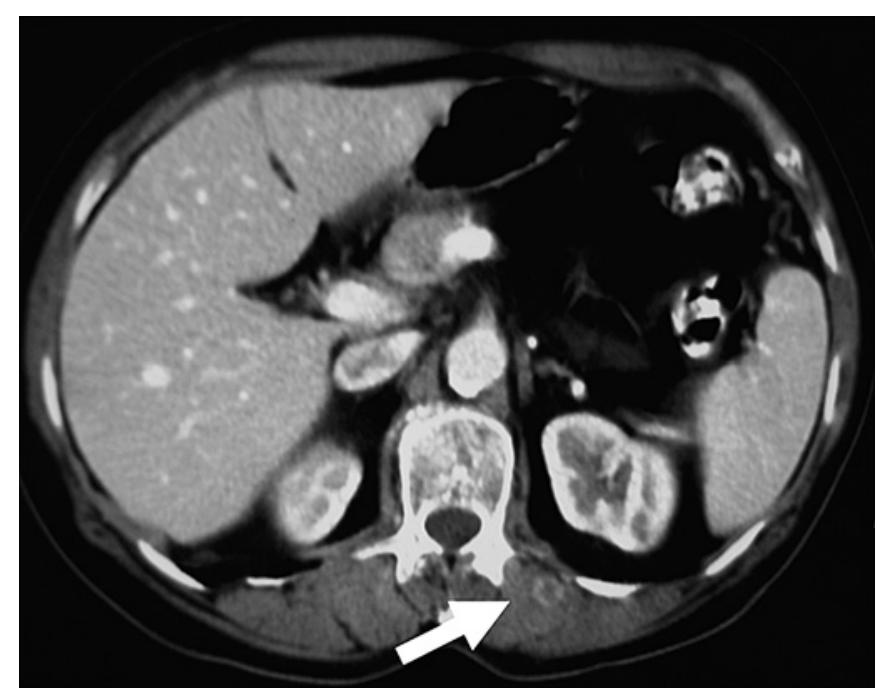

Figure 2 CT scan demonstrating a round-shaped soft tissue mass in the left paraspinal muscle (arrow).

\section{Learning points}

- Despite its rich vasculature, skeletal muscle has a relative resistance to haematogenous metastases. ${ }^{1}$ Muscle metastasis represents less than $1 \%$ of all metastases from solid tumours. The most common primary neoplasm localisation is lung.

- Metastatic disease to muscle presents as a painful mass, increased muscle tone and swelling. Hence, asymptomatic muscle metastasis is a rare clinical presentation of metastatic lung cancer.

- A combined FDG-PET/CT scan has greater sensitivity than a CT scan in the evaluation of extension of disease in lung cancer patients. ${ }^{2}$

- The differential diagnosis of a solitary FDG abnormal uptake in muscle metastasis includes synchronous tumour (either benign or soft tissue sarcoma), abscess, parasites, hibernoma, haematoma, trauma and foreign body. Thus, pathological confirmation of the nature of FDG muscle uptake should be considered mandatory.

positron emission tomography (FDG-PET)/CT scan for staging demonstrated hypermetabolic FDG uptake in the lung mass (figure 1A) and an unexpectedly focal FDG uptake in the left paraspinal muscle (figure 1B). Physical examination was normal. Biopsy from the muscle showed infiltration with cells of adenocarcinoma. A revision of the initial CT scan revealed a mass in the left paraspinal muscle which had not been reported (figure 2, arrow).

\section{Ignacio Banzo, Isabel Martínez-Rodriguez, Remedios Quirce,} Julio Jimenez-Bonilla, Jose M Carril

Servicio de Medicina Nuclear, Hospital Universitario Marqués de Valdecilla, Universidad de Cantabria, Santander, Spain

Correspondence to Dr Ignacio Banzo, Facultad de Medicina, Universidad de Cantabria, Av. Valdecilla s/n, 39008 Santander, Spain; mnubmj@humv.es

Competing interests None.

Patient consent Obtained.

Ethics approval This study was conducted with the approval of the Ethics Committee of Hospital Universitario Marqués de Valdecilla.

Provenance and peer review Not commissioned; externally peer reviewed.

Accepted 6 January 2011

Published Online First 9 February 2011

Thorax 2011;66:642. doi:10.1136/thx.2010.155903

\section{REFERENCES}

1. Seely S. Possible reasons for the high resistance of muscle to cancer. Med Hypotheses 1980;6:133-7.

2. Maziak DE, Darling GE, Inculet Rl, et al. Positron emission tomography in staging early lung cancer. A randomized trial. Ann Intern Med 2009;151:221-8. 